\title{
Evaluación de la factibilidad de mercado para la creación de una empresa
}

\section{Evaluation of the market feasibility for the creation of a company}

\author{
Miguel Ángel Reyes Hernández ${ }^{1}$, Dr. Germán Martínez Prats² , Dra. Francisca Silva
} Hernández ${ }^{3}$

\section{${ }^{1}$ Universidad Juárez Autónoma de Tabasco, México}

29miguelangelrey@gmail.com

https://orcid.org/0000-0002-0728-1007

\section{${ }^{2}$ Universidad Juárez Autónoma de Tabasco, México germanmtzprats@ hotmail.com \\ ORCID: https://orcid.org/0000-0001-6371-448X}

${ }^{3}$ Universidad Juárez Autónoma de Tabasco, México fany987@ hotmail.com

ORCID: https://orcid.org/0000-0003-3533-0002

\section{Dol https://doi.org/10.46589/rdiasf.vi35.370}

Recibido 18 de febrero 2021.

Aceptado 10 de abril 2021

Publicado 30 de junio de 2021

\section{Resumen}

Los aparatos electrodomésticos como procesos tecnológicos y de innovación requieren de un mantenimiento preventivo y de condición óptima para un mejor servicio de calidad. Por ello, evaluar la factibilidad de mercado que tiene una empresa dedicada al mantenimiento preventivo a través de la asistencia técnica, y acondicionamiento de electrodomésticos de línea blanca que han sido desechados requiere de aspectos sustanciales como crear una conciencia social en las personas respecto a los servicios de mantenimiento preventivo y acondicionamiento de electrodoméstico en este caso de línea blanca, determinar un mercado específico de la demanda de los servicios a partir de identificar los principales problemas que generan los electrodomésticos que han sido desechados y así detectar la factibilidad de mercado de este tipo de empresas.

Palabras clave: Factibilidad de mercado, servicio, sostenibilidad. 


\section{Abstract}

Household appliances as technological and innovation processes require preventive maintenance and optimum condition for a better quality service. Therefore, evaluating the market feasibility of a company dedicated to preventive maintenance through technical assistance, and conditioning of discarded white goods requires substantial aspects such as creating a social awareness in people regarding services of preventive maintenance and conditioning of household appliances in this case of white goods, to determine a specific market of the demand for services from identifying the main problems generated by household appliances that have been discarded and thus to detect the market feasibility of this type of Business.

Key words: Market feasibility, service, sustainability.

\section{Introducción}

La contaminación hoy en día está en un punto muy crítico, con la industrialización día con día salen productos nuevos y mejores de fácil implementación, así mismo sucede con los electrodomésticos que se usan en los hogares, aunque no son tan frecuentes como otros artículos, no dejan de convertirse en un contaminante más.

Muchas ocasiones los electrodomésticos son desechados (Oblitas et al, 2019) puesto que llegan a tener fallas ya sea por su constante uso, el poco cuidado que recibe o por dejarlos expuesto a la intemperie. Otros motivos pueden ser, su mala apariencia tras muchos años, llegan a ser anticuados, obsoletos o sustituidos por un electrodoméstico nuevo.

Para reducir la emisión de esta clase de contaminantes, se considera importante crear empresas dedicadas a la asistencia técnica y acondicionamiento de los electrodomésticos de línea blanca en el que todo hogar hay.

En la actualidad la línea de separación entre la electrónica y la electricidad es ligera, demasiado pequeña, por no decir nula, por lo tanto, de manera general se define como aparato o equipo eléctrico y electrónico a todo aquel que funcione adecuadamente a base de electricidad como fuente de energía. Estos aparatos cuando dejan de ser utilizados porque han cumplido con su ciclo de vida útil para una necesidad determinada, pasan a constituirse como todo en la vida en elementos llamados residuos o desechos cuya nomenclatura en español es RAEE-Residuos de Aparatos Eléctricos y Electrónicos o en inglés WEEE-Waste Electrical and Electronical Equippament (Hidalgo, 2010). 
La disposición de los RAEE se ve enfrentada a dos retos mayores: por un lado, lograr el mayor aprovechamiento de los materiales que se pueden recuperar de ellos; por otro lado, el manejo correcto de sustancias peligrosas, para garantizar el menor impacto a la salud y al ecosistema (Rodríguez et al, 2013).

Para esto es pertinente realizar un estudio de mercado en el cual se puedan demostrar la clasificación de productos y servicios, la importancia que este servicio tiene, así como la demanda que se podrá determinar de acuerdo a los estudios e investigaciones primarias y secundarias, de este modo también se puede considerar el tipo de oferta que se realiza al mercado que se desea enfocar teniendo la idea del tipo de comercialización que se puede llevar a cabo. Por tanto, Fernández (2014) entiende por obsolescencia programada la fecha de caducidad impuesta por los fabricantes para cortar el ciclo de vida de sus productos, en este caso los aparatos electrónicos, como los productos comerciales que tienen un ciclo de vida desde su puesta en el mercado hasta su obsolescencia, rotura y caducidad.

\section{Conceptos y técnicas}

En la actualidad existen empresas que ofrecen bienes y servicios para todo tipo de necesidades y gustos, es muy común encontrar servicios y productos que pueden llegar a ser demasiados costosos, a como también podemos encontrar productos y servicios que pueden ser a precios muy accesibles, todos tienen como fin satisfacer una necesidad.

Se puede encontrar productos con precios realmente costosos que para la mayoría de las personas que tienen ingresos promedios, sería casi imposible adquirir, pero hay personas que puedan llegar a pagar el costo, los productos están dirigidos a cierto tipo de personas, ya que todo es referente a la segmentación de mercado, o de una manera más simple, común y muy conocida manera de decirlo sería, la clase social.

Para poder lanzar cualquier producto y/o servicio siempre ha sido necesario determinar al tipo de persona o segmentación de mercado a la que uno se quiero dirigir, para esto es necesario realizar una investigación de mercado. Pero, ¿Qué es una investigación de mercado? Según Baca (2010) "la investigación de mercados proporciona información que sirve de apoyo para la toma de decisiones, la cual está encaminada a determinar si las condiciones del mercado no son un obstáculo para llevar a cabo el proyecto" entonces se puede definir que una investigación de 
mercado es un conjunto de acciones que tienen como objetivo recopilar información precisa, oportuna y relevante, ayudando a crear una opinión en las decisiones para la creación de una empresa o negocio.

\section{- Cómo determinar la factibilidad}

Las empresas y negocios surgen de una idea, para poder llevar esta idea a cabo, se recomienda tener distintos puntos de vista, ya que al considerarlas se puede prevenir y disminuir riesgos a los que puedes estar expuesto al momento de la creación de tu empresa, esta técnica se conoce como evaluación de proyecto, la misma técnica cuenta con la herramienta que se conoce como estudio de factibilidad, la cual se encarga de poder determinar distintos aspectos en la creación de la empresa, el estudio se enfoca en los puntos siguientes:

- Análisis del mercado

- Análisis técnico operativo

- Análisis económico financiero

- Análisis socioeconómico

Las cuales tiene como finalidad poder aportar la información necesaria que puedan servir a la toma de daciones al momento de crear la empresa, sin embargo, para este trabajo de investigación nos enfocaremos en el análisis del mercado.

Asimismo, dentro del campo teórico la utilidad del artículo se encuentra en la utilización de los conceptos de finanzas para estudios de factibilidad y competitividad. Se aplican principalmente los conceptos de viabilidad del proyecto basados en los criterios de Tasa Interna de Rentabilidad y Valor Presente Neto, e igualmente se hace referencia al concepto de planeación estratégica organizando la información de la industria en el modelo de las cinco fuerzas desarrollado por Michael Porter, para mostrar la factibilidad del proyecto (Cuevas y Moreno, 2003).

- Análisis del poder de los competidores

- Análisis del poder de los compradores

- Análisis del poder de los proveedores

- Productos sustitutos

- Barreras de entrada 


\section{- $\quad$ Análisis de mercado}

El análisis del mercado es el principal aspecto que se debe considerar para la creación de una empresa, puesto que es el principal punto para suponer si la creación de una empresa realmente será factible.

Según Baca Urbina (2010) para determinar una factibilidad del mercado es necesario realizar un estudio de la oferta y demanda que existe de acuerdo al servicio que se pretende ofrecer, para realizar un estudio de la demanda será necesario recabar información secundaria que nos pueda orientar para saber si el servicio que pretendemos ofrecer ya existe y si ha sido aceptado, de no haber un estudio previo, se recomienda realizar un estudio primario, no hay nada mejor que recabar información confiable y veraz que de la principal fuente, para esto será necesario definir objetivos de lo que realmente nos interesa saber, se determinara una muestra de la población a la que nos vamos dirigir, de acuerdo a los objetivo y la muestra, se diseñará una encuesta o entrevista que nos ayudará a recabar los datos necesarios, se pasará al proceso de análisis para poder comprender y determinar si el estudio que se realizó realmente cumple con la validez y confiabilidad, de no ser así se deberá restructurar un nuevo instrumento de investigación, si los resultados no son favorables se tomará en consideración volver aplicarlo para una segunda opinión, sin los resultados siguen sin ser favorable se puede abandonar la investigación, ya que los demás puntos no serían necesarios si no hay un demanda, mas sin embargo se podrá optar por implementar un fuerte campaña de marketing que ayude en aceptación del servicio que se ofrece.

\section{- $\quad$ Como hacer un estudio de mercado}

Para la realización de un estudio de mercado será necesario tener en cuenta diferente factores, ya que influyen en la toma de decisiones para la creación de un proyecto de negocio, según Benassini (2009) "se requiere de información relevante acerca del mercado y de sus competidores, que le permita tomar decisiones más acertadas ante los diferentes ambientes económicos, políticos y sociales que cambian cada vez con mayor velocidad" tomando en consideración que se debe visualizar los distintos puntos que se requiere para un estudio de mercado, según Sapag (2001) la etapa de idea es el proceso de búsqueda que surge de haber identificado posibles soluciones a un problema a través de un análisis en los procesos o de realizar cambios en etapas ineficientes, dando como resultado oportunidad de mejoramiento.

De acuerdo a varios autores se realizan varias etapas para el estudio de mercado 
1. Formulación de problema: es la primera parte para dar inicio al estudio de mercado, ya que en este punto especifican de una manera clara cuáles serán los objetivos de la investigación.

2. Fuentes de información: siempre es necesario tener información que nos oriente, ya que nos da una mayor visón a lo que necesitamos saber, para tal caso se contempla que hay dos fuentes de información, las fuentes primarias y secundarias, la fuente primaria son los resultados directos que hemos obtenido tras realizar una investigación de campo y la fuente secundaria es la información generada por alguien más, pude ser recopilada de distintas fuentes, libros, enciclopedias, artículos, páginas de internet, revistas. Toda aquella información que pueda servir para la investigación,

3. Diseño de muestra: ya que no se puede entrevistar o encuestar a toda la población, es necesario que se determine la muestra, que se refiere a dirigirse a ciertas personas contemplando un porcentaje de toda la población.

4. Instrumento de investigación y recopilación de datos: se determina el instrumento que mejor nos ayude a recolectar la información que necesitamos, de acuerdo a la información que se necesita el instrumento puede variar, puede ser una encuesta, una entrevista, una observación de campo, cedula de investigación

5. Análisis de los datos recabados: una vez aplicado los instrumentos de investigación se llevará a cabo el análisis de los resultados que se obtuvieron, mediante la revisión y codificación.

6. Presentación del informe: al haber realizado la recolección de los datos se realiza un informe en el cual se manifiestan los resultados obtenidos de manera oportuna.

\section{Determinación de la demanda}

Un análisis de la demanda es el principal objetivo de realizar un estudio de mercado, para esto debemos comprender en que consiste la demanda y como poder determinar la capacidad de la demanda. De acuerdo a Baca Urbina (2010) la demanda es la cantidad de bienes y servicios que el mercado requiere o solicita para buscar la satisfacción de una necesidad específica a un precio determinado. Para poder comprender como determinar la demanda.

Según Sapag (1991) en términos generales, la cantidad de la demanda de un bien aumenta al bajar el precio del producto, al aumentar el precio de los bienes 
sustitutos 0 al reducirse el de los complementarios, al aumentar el ingreso del consumidor y al aumentar las preferencias del consumidor por ese producto.

Para esto es necesario poder comprender la teoría de la demanda en la cual explica que al subir el precio de los productos la demanda baja ya que no logran tener el poder adquisitivo, de manera inversa, al bajar el precio de los productos la demanda aumenta ya que tendrá mayor poder adquisitivo para poder realizar la compra.

Para tal caso se deberá realizar un estudio socioeconómico en el cual se pueda determinar el poder adquisitivo de las personas a las que nos pretendemos acercar para ofrecer los servicios.

\section{Conclusión}

Al determinar la factibilidad del mercado para la creación de una empresa de servicios técnicos y rehabilitación de electrodomésticos de línea blanca desechados habrá una concientización en las personas al fomentar la cultura de rehabilitar así como el de reciclar, dándoles a conocer que a sus electrodomésticos se les puede extender su vida útil mediante los servicios de asistencia técnica preventiva, correctiva y de rehabilitación, así mismo al aplicar los servicios ya mencionado, se podrá conseguir una disminución considerable en la emisión de estos electrodomésticos como desechos contaminantes.

\section{Referencia}

Baca Urbina, Gabriel. (2010). Evaluación de proyectos. México: McGRAW-HILL.

Benassini, Marcela. (2009). Introducción a la investigación de mercados: enfoque para América Latina. México: PEARSON EDUCACIÓN.

Cuevas Villegas, Carlos Fernando \& Moreno Lasprilla, Freddy Hernán. (2003). Estudio de factibilidad para la creación de una empresa de servicios de ingeniería. Revista Estudios gerenciales, 19(88), 33-48.

Fernández Rey, Laura. (2014). La obsolescencia programada: sus consecuencias en el ambiente y la importancia del consumo responsable. Revista Terra Mundus, 1(1): 1-12. 
Hidalgo Aguilera, Luis. (2010). La basura electrónica y la contaminación ambiental. Revista Enfoque UTE, 1(1), 46-61.

Oblitas Cruz, Jimy Frank; Sangay Terrones, Max Edwin; Rojas de la Puente, Edward Enrique \& Castro Silupu, Wilson Manuel. (2019). Economía circular en residuos de aparatos eléctricos y electrónicos. Revista de Ciencias Sociales, XXV (2), 196-208.

Rodríguez B., Luz Angélica, González E., Nicolás, Reyes R., Lorena S., \& Torres R., Andrés F. (2013). Sistema de gestión de residuos de aparatos eléctricos y electrónicos. Enfoque de dinámica de sistemas. Revista Sistemas \& Telemática 11(24), 39-53.

Sapag Chain, Nassair. (2001). Evaluación de proyectos de inversión en la empresa. Argentina: PEARSON EDUCATION.

Sapag Chain, Nassir \& Sapag Chain, Reinaldo. (1991). Preparación y evaluación de proyectos. México: McGRAW-HILL.

Cómo citar este artículo:

Martínez Prats, G., Reyes Hernández, M., \& Silva Hernández, F. . (2021). Evaluación de la factibilidad de mercado para la creación de una empresa. Revista De Investigación Académica Sin Frontera: División De Ciencias Económicas Y Sociales, (35). https://doi.org/10.46589/rdiasf.vi35.370

https://revistainvestigacionacademicasinfrontera.unison.mx/index.php/RDIASF/article/view/3 $\underline{70}$

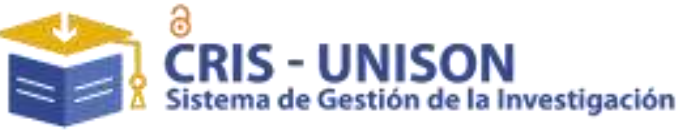

\section{Dialnet}

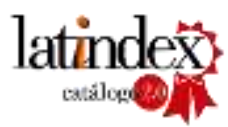

[If InAcron REDIB

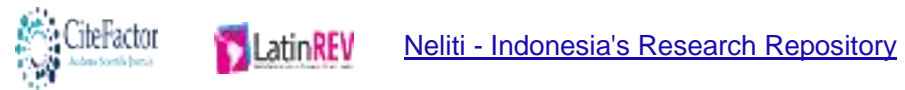
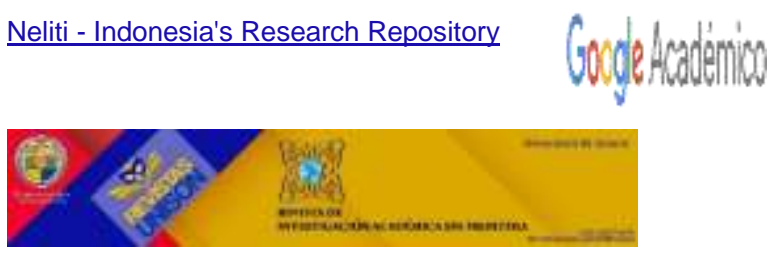